\title{
Evaluation of multiple protein docking structures using correctly predicted pairwise subunits
}

\author{
Juan Esquivel-Rodríguez ${ }^{1}$, Daisuke Kihara ${ }^{1,2^{*}}$ \\ From Great Lakes Bioinformatics Conference 2011 \\ Athens, OH, USA. 2-4 May 2011
}

\begin{abstract}
Background: Many functionally important proteins in a cell form complexes with multiple chains. Therefore, computational prediction of multiple protein complexes is an important task in bioinformatics. In the development of multiple protein docking methods, it is important to establish a metric for evaluating prediction results in a reasonable and practical fashion. However, since there are only few works done in developing methods for multiple protein docking, there is no study that investigates how accurate structural models of multiple protein complexes should be to allow scientists to gain biological insights.

Methods: We generated a series of predicted models (decoys) of various accuracies by our multiple protein docking pipeline, Multi-LZerD, for three multi-chain complexes with 3, 4, and 6 chains. We analyzed the decoys in terms of the number of correctly predicted pair conformations in the decoys.

Results and conclusion: We found that pairs of chains with the correct mutual orientation exist even in the decoys with a large overall root mean square deviation (RMSD) to the native. Therefore, in addition to a global structure similarity measure, such as the global RMSD, the quality of models for multiple chain complexes can be better evaluated by using the local measurement, the number of chain pairs with correct mutual orientation. We termed the fraction of correctly predicted pairs (RMSD at the interface of less than 4.0Å) as fpair and propose to use it for evaluation of the accuracy of multiple protein docking.
\end{abstract}

\section{Introduction}

An essential part of protein structure prediction is to establish methods to evaluate computed models. For single protein structure prediction, the research community, which is partly driven by the Critical Assessment of Protein Structure Prediction (CASP), often uses the global RMSD as well as its variant, the GDT-TS score [1]. On the other hand, the protein docking community, which is partly led by the Critical Assessment of Prediction of Interactions (CAPRI) [2], often uses the RMSD at the docking interface named the iRMSD and the fnat (fraction of correctly predicted native contacts). The iRMSD and the fnat are originally designed to evaluate the accuracy of pairwise protein docking models.

\footnotetext{
* Correspondence: dkihara@purdue.edu

'Department of Computer Science, College of Science, Purdue University, West Lafayette, IN 47907, USA

Full list of author information is available at the end of the article
}

Earlier works on multiple protein docking used the global RMSD for evaluating the model accuracy [3-7]. Of course the global RMSD, the iRMSD, or the fnat can be used to identify accurate models of multiple chain complexes. However, since the sizes of the whole multiple chain complexes can be much larger than single protein structures or pairwise protein complexes, the usefulness of multiple chain complex models can be better understood and evaluated if the global quality measures are complemented by additional measures that quantify local accuracy of models.

Here, we generated decoys of multiple protein complexes using Multi-LZerD [8,9], a multiple protein docking method developed in our group. We analyzed the decoys in terms of the number of pairwise interactions in whole multiple chain complexes that have been accurately predicted, that is, pairs with an iRMSD of less than $4 \AA$ (the iRMSD only takes into account atomic coordinates at the 
docking interface region). We show that, even when the apparent overall RMSD of a multi-chain complex seems to be high, in many cases there are several accurately predicted pairwise interactions. Such models would be still useful for certain purposes since they contain a significant number of docking interface residues that are correctly placed relative to their interacting chains. We highlight this by proposing a new accuracy measure for multiple docking, named fpair (fraction of pairwise hits) that accounts for the proportion of correct pairwise predictions among all chain pairs in a whole multiple chain complex.

\section{Methods}

We used Multi-LZerD [8,9] to construct decoys of various global accuracy (RMSD) ranges for three multiple protein complexes: $1 \mathrm{~A} 0 \mathrm{R}, 1 \mathrm{NNU}$, and $1 \mathrm{I} 3 \mathrm{O}$, which are 3,4 , and 6 chain complexes. Here we briefly explain the Multi-LZerD algorithm. Multi-LZerD takes the 3D structure of component chains of a multiple chain complex as input, and first employs the LZerD algorithm [10], a pairwise protein docking method developed in our group, to generate a few tens of thousands pairwise docking conformations for each chain pair. A characteristic of LZerD is that it uses the 3D Zernike descriptors $[11,12]$, a series expansion of a 3D function, to represent protein surface shape and to identify shape complementarity of surfaces.

A conformation of a multiple chain complex can be uniquely specified by denoting which pairwise docking decoys to combine from the pool of the pre-computed pairwise decoys by LZerD. Multi-LZerD explores different conformations of the whole complex by altering pairwise decoys using a genetic algorithm [13]. The fitness function used to evaluate decoys is a linear combination of physics-based scoring terms. After 3000 generations, Multi-LZerD finally outputs 200 models of the complex. Clustering is applied at the end of every generation [14], thus, the number of final set of decoys is less than 200 .

\section{Results}

\section{Correct pairwise interactions in decoys}

Three protein complexes of different number of chains, 3 (PDB ID: 1A0R), 4 (1NNU), 6 (1I3O) were used in this study. These decoys are classified by the RMSD into

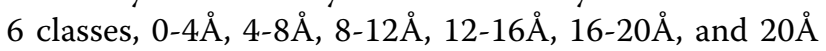
or larger. Additionally, for each decoy, we computed the iRMSD for all pairs of chains included in the complex by comparing each pair in the decoy to the corresponding pair in the native structure. If the iRMSD is lower than $4 \AA$ the pair is considered a hit. An iRMSD of $4 \AA$ is a criterion of acceptable prediction for pairwise docking used in the CAPRI. Using the pairwise hit count in a whole complex decoy we calculate the fpair value, which is defined as the fraction of pairwise combinations that are considered hits, from the total pairwise combinations:

$$
\text { fpair }=\frac{\sum_{p \in P} I(i R M S D(p)<4 \text { Angstrom })}{\left(\begin{array}{l}
N \\
2
\end{array}\right)},
$$

where $P$ is the set of all pairwise combinations. $I$ is the indicator function that represents 1 if the predicate is true (i.e. iRMSD is smaller than $4 \AA$ ) and 0 otherwise.

For example, a 3-chain complex with chains $\mathrm{A}, \mathrm{B}$, and $\mathrm{C}$ has 3 pairwise combinations, $\mathrm{A}-\mathrm{B}, \mathrm{A}-\mathrm{C}$, and $\mathrm{B}-\mathrm{C}$. A-B from a predicted structure is superimposed onto $A-B$ structure taken from the native complex structure, without taking the chain $\mathrm{C}$ into account. The same process is repeated for A-C and B-C. If 2 out of these 3 pairwise combinations are hits then fpair is $2 / 3=0.67$ for the predicted complex structure.

Figure 1 shows the distribution of the global RMSD of the decoys classified into the 6 classes and the number of the pairwise hits included in the decoys. Figure 1A shows the results for $1 \mathrm{~A} 0 \mathrm{R}$, a 3 chain complex. There was one decoy in the range of $0-4 \AA$ (leftmost bar). For this decoy, all 3 chain pairs have iRMSD $<4 \AA$. No decoys are found in the 4-8 $\AA$ global RMSD range. Interestingly, all the decoys in the range of $8-12 \AA$ ( 6 decoys), $12-16 \AA$ (6 decoys), $16-20 \AA$ ( 23 decoys) and $20 \AA+$ range (86 decoys) have one pairwise hit, which gives an fpair of 0.33 . Thus, even when the global RMSD is very large, e.g. $20+\AA$, 2 out of 3 chains are correctly predicted and the location of additional one chain made the apparent global RMSD large.

Figure $1 \mathrm{~B}$ shows the results for $1 \mathrm{NNU}$, a 4 chain complex (thus there are $6=4 \times 3 / 2$ chain pairs). Out of 10 decoys in the global RMSD range of 12-16 $\AA$, 4 decoys contain 2 pairwise hits (fpair of 0.33 ) while the other 6 decoys contain 3 pairwise hits (fpair of 0.5). Even in higher global RMSD ranges, there are still chain pairs that are correctly predicted. In the range of $16-20 \AA$ there are 2 decoys with 2 pairwise hits and 35 with 3 hits. Finally, in the $20 \AA$ + range 10 decoys have 2 hits, 74 decoys have 3 hits and 1 decoy has 4 pairwise hits (fpair 0.67).

Figure $1 \mathrm{C}$ presents the results for $1 \mathrm{I} 3 \mathrm{O}$, a 6 chain complex. In the range of $0-4 \AA$, all 5 decoys have the maximum number of pairwise hits, 15 , i.e. a fpair value of 1.0. In the next three classes, $4-8 \AA, 8-12 \AA$, there are significant number of decoys with 12 and 11 hits, which yield fpair of 0.8 and 0.73 , respectively. At the range of 12-16 $\AA$, there is one decoy with 7 hits (fpair of 0.47 ). Finally, it is notable that the last 2 classes, $16-20 \AA$ and $20+\AA$, are dominated by decoys with 11 hits, except for 3 decoys in the $20 \AA$ + range that have 7 hits. 

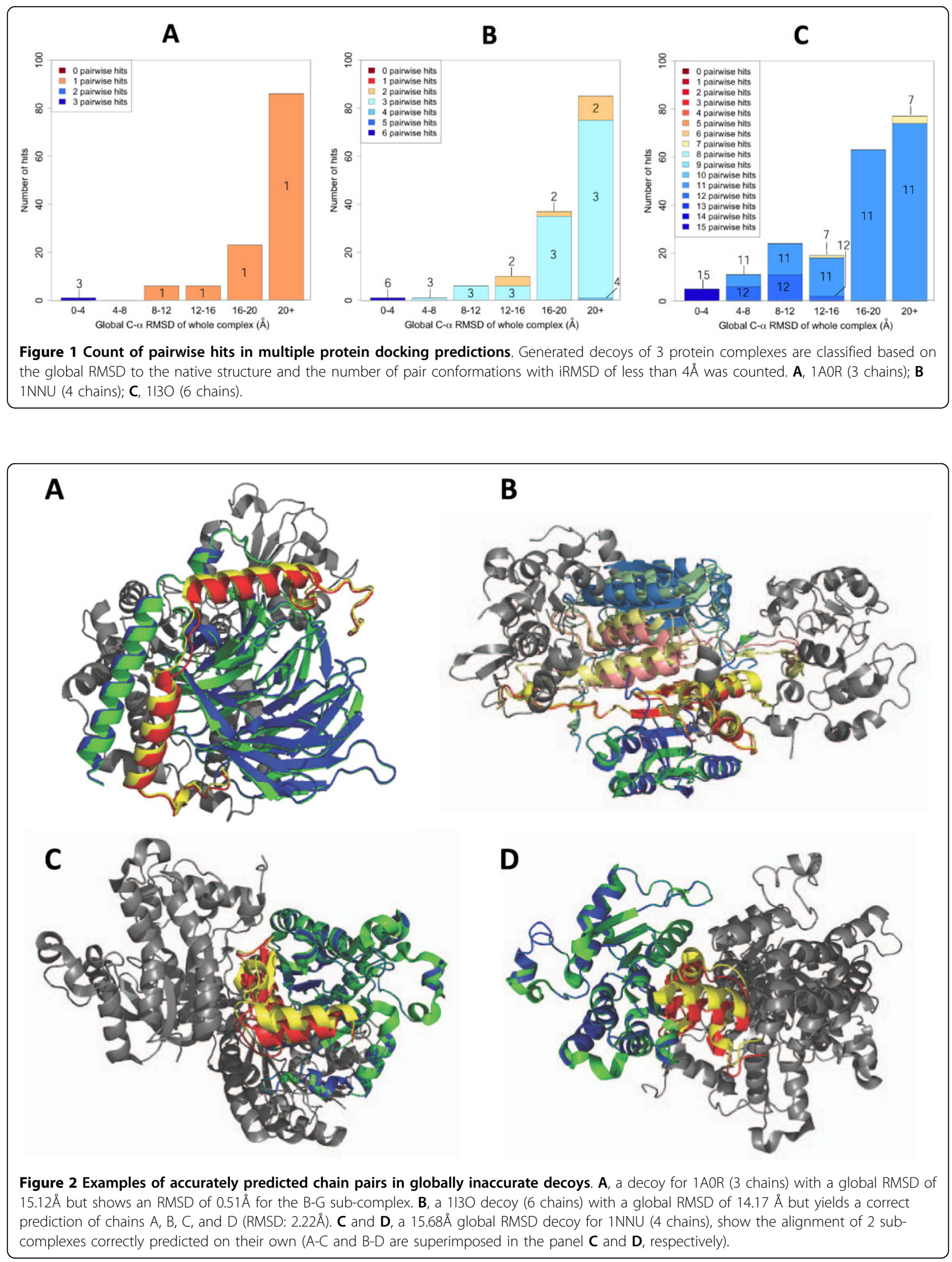


\section{Examples of decoy structures}

In Figure 2 we show examples of decoys with a high global RMSD but contain correctly predicted chain pair conformations. Figure 2A shows a decoy for 1A0R, of which global RMSD is $15.12 \AA$. Despite of the seemingly large RMSD, relative positions of two chains B (green/ blue for native/predicted position of the chain) and $G$ (yellow/red for native/predicted) are very well predicted with an iRMSD of $0.51 \AA$. A similar case can be seen in Figure $2 \mathrm{~B}$, where a decoy for $1 \mathrm{I} 3 \mathrm{O}$ is presented. The global RMSD of this decoy is $14.17 \AA$; however, relative positions of 4 chains out of 6 chains, chains A (green/ blue for native/predicted), B (yellow/red), C (pale green/ pale blue), and D (pale yellow/pale red) are very well predicted with a RMSD of $2.22 \AA$. Finally, Figures $2 \mathrm{C}$ and 2D show a decoy for $1 \mathrm{NNU}$ (15.68 $\AA$ global RMSD). The decoy is shown from two different angles to clearly show the pairs of chains A-C and B-D, both of which are predicted within iRMSD of $4.0 \AA$.

\section{Conclusions}

We have shown that, while the global C- $\alpha$ RMSD is a clear indication of high quality predictions for multiple protein docking, a predicted structure with a higher RMSD should not be simply discarded as unsuccessful prediction since in many cases such decoys contain correctly predicted subcomplexes. We propose a measure named fpair for assessing the fraction of correctly predicted pairs among all pairs in a whole complex. By using fpair one can distinguish models that have partially accurate subcomplexes from models with the same global RMSD but do not contain any correctly predicted pairs. fpair will complement the traditional global measurements like RMSD and fnat for evaluating quality of models for multiple protein complexes.

\section{Acknowledgements}

This work was supported by the National Institute of General Medical Sciences of the National Institutes of Health [R01GM075004 to DK] and the National Science Foundation [DMS0800568, EF0850009, IIS0915801 to DK]. JER is a Fulbright Science and Technology Fellow.

This article has been published as part of BMC Bioinformatics Volume 13 Supplement 2, 2012: Proceedings from the Great Lakes Bioinformatics Conference 2011. The full contents of the supplement are available online at http://www.biomedcentral.com/bmcbioinformatics/supplements/13/S2

\section{Author details}

'Department of Computer Science, College of Science, Purdue University, West Lafayette, IN 47907, USA. ²Department of Biological Sciences, College of Science, Purdue University, West Lafayette, IN 47907, USA.

\section{Authors' contributions}

JER developed the multiple protein docking prediction method, Multi-LZerD, performed the computational experiments and wrote the manuscript draft. DK conceived the study and participated in its design and coordination, as well as drafting and finalizing the manuscript. All authors read and approved the final manuscript.

\section{Competing interests}

The authors declare that they have no competing interests.

Published: 13 March 2012

\section{References}

1. Zemla A: LGA: A method for finding 3D similarities in protein structures. Nucleic Acids Research 2003, 31:3370-4

2. Janin J: Protein-protein docking tested in blind predictions: the CAPRI experiment. Molecular BioSystems 2010, 6:2351-62.

3. André I, Bradley P, Wang C, Baker D: Prediction of the structure of symmetrical protein assemblies. Proceedings of the National Academy of Sciences of the United States of America 2007, 104:17656-61.

4. Berchanski A, Eisenstein M: Construction of molecular assemblies via docking: modeling of tetramers with D2 symmetry. Proteins 2003, 53:817-29.

5. Comeau SR, Camacho CJ: Predicting oligomeric assemblies: N-mers a primer. Journal of Structural Biology 2005, 150:233-44.

6. Inbar $\mathrm{Y}$, Benyamini $\mathrm{H}$, Nussinov $\mathrm{R}$, Wolfson $\mathrm{HJ}$ : Prediction of multimolecular assemblies by multiple docking. Journal of Molecular Biology 2005, 349:435-47.

7. Karaca E, Melquiond ASJ, Vries SJ de, Kastritis PL, Bonvin AMJJ: Building macromolecular assemblies by information-driven docking: introducing the HADDOCK multi-body docking server. Molecular \& Cellular Proteomics: MCP 2010, 9:1784-94.

8. Esquivel-Rodríguez J, Yang YD, Kihara D: Multiple protein docking for asymmetric hetero oligomeric complexes (poster). The 21st International Conference on Genome Informatics Hangzhou, China; 2010.

9. Esquivel-Rodríguez J, Yang YD, Kihara D: Multiple protein docking prediction method which can handle asymmetric hetero oligomeric complexes (poster). 3DSIG 2010: The 6th Structural Bioinformatics and Computational Biophysics Meeting Boston, USA; 2010.

10. Venkatraman V, Yang YD, Sael L, Kihara D: Protein-protein docking using region-based 3D Zernike descriptors. BMC Bioinformatics 2009, 10:407.

11. Novotni M, Klein R: 3D zernike descriptors for content based shape retrieval. Proceedings of the Eighth ACM Symposium on Solid Modeling and Applications - SM '03 2003, 216.

12. Sael $L$, Kihara D: Protein surface representation and comparison: new approaches in structural proteomics. In Biological Data Mining. Boca Raton, FL: Chapman \& Hall/CRC;Chen JY, Lonardi S 2009:89-109.

13. Raidl GR, Julstrom BA: Edge sets: an effective evolutionary coding of spanning trees. IEEE Transactions on Evolutionary Computation 2003, 7:225-239.

14. Comeau SR, Gatchell DW, Vajda S, Camacho CJ: ClusPro: an automated docking and discrimination method for the prediction of protein complexes. Bioinformatics 2004, 20:45-50.

doi:10.1186/1471-2105-13-S2-S6

Cite this article as: Esquivel-Rodríguez and Kihara: Evaluation of multiple protein docking structures using correctly predicted pairwise subunits. BMC Bioinformatics 2012 13(Suppl 2):S6.

\section{Submit your next manuscript to BioMed Central and take full advantage of:}

- Convenient online submission

- Thorough peer review

- No space constraints or color figure charges

- Immediate publication on acceptance

- Inclusion in PubMed, CAS, Scopus and Google Scholar

- Research which is freely available for redistribution 